\title{
Study on Applying Oxidized Poly-silicic-ferric Coagulant to Combined Process of Short-term Coagulation and Filtration
}

\author{
GAO Dong ${ }^{a}$, FU Ying ${ }^{*}$ \\ School of Civil Engineering and Architecture, University of Jinan, Jinan 250022, China \\ a963375043@qq.com, bcea_fuy@ujn.edu.cn
}

\begin{abstract}
Keywords: Oxidized poly-silicic-ferric; short-term coagulation; sedimentation; filtration
Abstract. The study investigated the effect of coagulant dosage, flocculation time, and precipitation time on the turbidity removal rate of Oxidized Poly-silicic-ferric (OPSF) coagulant compared with the polyferric aluminum (PFA) coagulant. The effect of OPSF on coagulation and filtration under short-time coagulation conditions was also studied. Experiments showed that when the optimum dosage was $6 \mathrm{mg} / \mathrm{L}$, the flocculation time had a significant influence on the turbidity removal rate of OPSF. With the increase of precipitation time, the turbidity removal effect of OPSF was relatively stable, which was about $94 \%$. The OPSF short-term coagulation and ordinary coagulation have the same effect on the turbidity removal effect, but PFA was different. And OPSF "short-time coagulation and filtration" was better than PFA "ordinary coagulation and filtration".
\end{abstract}

\section{Introduction}

Coagulation is the process that small particles (including colloids and suspended solids) that are difficult to aggregate and precipitate in water aggregate into larger particles and be removed by physical and chemical processes. The coagulation effect has a profound influence on the subsequent treatment processes, and the hydraulic conditions of coagulation are one of the direct factors influencing coagulation effect. In practice, the coagulation and sedimentation occupy a larger area due to the need of the treatment effect. In recent years, researches and applications of high-efficiency water treatment processes that shorten process flow have been carried out in China and foreign countries. In order to settle most of flocs, the coagulation time of 21-62 min in conventional coagulation [1-4]. However, loading flocculation used by Chen Yuquan et al. who found that flocs precipitate rapidly within $10 \mathrm{~min}$ [5]. In this paper, the coagulation-sedimentation process of approximately 21-62 min is called ordinary coagulation, while of not more than $10 \mathrm{~min}$ is called short-time coagulation. Typical high-efficiency coagulation-sedimentation process is characterized by its high efficiency, rapid settling speed, and small occupied area, such as Densadeg high-density sedimentation tank of Degremont Company in France and Turbo-LME high-speed sedimentation tank of Paswing-Lotig Company in Germany [6]. The high-efficiency water treatment methods above are all optimized studies in terms of treatment process and reactor.

If it is possible to shorten the time of coagulation and sedimentation based on the advantages of high-performance coagulants, it would not only save land, but also solve the partially problems of complex construction, lack of operation and maintenance personnel and difficulty in centralized water supply resulted from traditional water treatment facilities in rural areas. So far, the study of "short-time coagulation and sedimentation" based on high-efficient coagulants has rarely been reported compared with ordinary coagulation.

OPSF is a high-efficiency inorganic polymer coagulant, which has strong charge neutralization, adsorption bridging and oxidation capacity [7]. The study based on the high-efficiency OPSF coagulant investigated the main flocculating time and sedimentation time, and the effect of the "short-term coagulation and filtration" combined process was also explored. It provides an experimental and theoretical basis for optimizing equipped and modular water treatment facilities and accelerating rural drinking water safety. 


\section{Materials and Methods}

Chemicals. Kaolin, humic acid and sodium hydroxide $(\mathrm{NaOH})$ were purchased from Shengkai Co., Ltd., Jinan, China. All chemicals were of analytical grade and used as received without further purification. Polyferric aluminum was obtained from Yuqing Water Plant, Jinan, China, which ferric-aluminum content of $10.26 \%$. Poly-silicic-ferric was homemade (preparation method refers to references [1]).

\section{Analytical Methods.}

Water Samples. The experimental water sample was simulated water of kaolin and humic acid. The simulated water was mixed by $1.0 \mathrm{~g} / \mathrm{L}$ of humic acid stock solution and kaolin stock solution called humic acid simulation water. The characteristics of humic acid simulation water are presented in Table 1.

Table 1 The characteristics of humic acid simulation water

\begin{tabular}{ccccc}
\hline Temperature $\left[{ }^{\circ} \mathrm{C}\right]$ & $\mathrm{pH}$ & Turbidity [NTU] & Chroma [A] & Permanganate index [mg/L] \\
\hline $21.4 \sim 28.2$ & $8.60 \sim 8.92$ & $25 \sim 35$ & $0.333 \sim 0.438$ & $7.67 \sim 9.7$ \\
\hline
\end{tabular}

Jar Tests. Coagulation experiments were performed on a six-pitch mixer (ZR4-6, Zhongrun Water Industry Technology Development Co., Ltd., Shenzhen, China). The coagulation experiment started with rapid mixing at $200 \mathrm{r} / \mathrm{min}$ for $1 \mathrm{~min}$ and a certain amount of coagulant was added at the beginning of the mixing. Then the stirring speed was reduced to $40 \mathrm{rpm}$ with a duration flocculation time of $15 \mathrm{~min}$. After sedimentation for $10 \mathrm{~min}$, a $100 \mathrm{~mL}$ water sample was taken from $4 \mathrm{~cm}$ below the liquid surface for measuring turbidity using a turbidimeter (2100AN, HACH Water Quality Analysis Instrument Co., Ltd., Shanghai, China).

"Short-time Coagulation and Filtration" Experiment. The coagulation and sedimentation procedure was operated according to above jar tests. The supernatant obtained after coagulation and sedimentation was transported into a filtration device (homemade) by peristaltic pump (BT100, Baoding Lange Constant Flow Pump Co., Ltd.,China). Filtration experiment was performed in filter column made of plexiglass (diameter $30 \mathrm{~mm}$ ). Quartz sand as filter material with thickness of $70 \mathrm{~cm}$, and cobblestones as supporting layer with thickness of $10-20 \mathrm{~cm}$. The initial filtration rate was set to 7 $\mathrm{m} / \mathrm{h}$. Water samples were taken every hour to measure the turbidity.

\section{Results and Discussion}

\section{Effect of Coagulant Dosage on Removal of}

Turbidity. This experiment was conducted to get the effect of coagulant dosage on turbidity removal under ordinary coagulation conditions. As shown in Fig. 1, the removal rates of turbidity were increased to $97 \%$ with OPSF dosage increased from $4 \mathrm{mg} / \mathrm{L}$ to 10 $\mathrm{mg} / \mathrm{L}$, then decrease slightly. However, given that the removal rates of turbidity increased fastest at 4-6 $\mathrm{mg} / \mathrm{L}$, this experiment selected $6 \mathrm{mg} / \mathrm{L}$ as OPSF optimum dosage. According to the comparison of the two curves, it can be seen that the difference between OPSF and

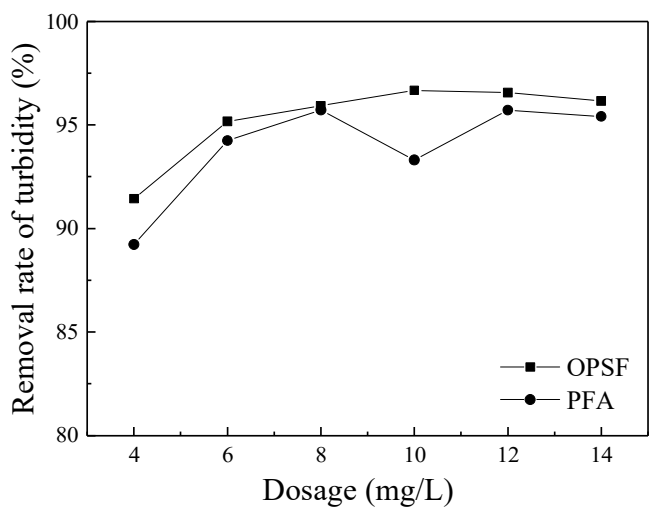

Fig. 1 Effect of Dosage on Removal of Turbidity PFA in removal rates of turbidity was small.

Determination of OPSF Short-term Coagulation Parameters. The flocculation time was adjusted from 2 to 12 min to investigate the influence of it on the turbidity removal rate of OPSF. OPSF optimum dosage was $6 \mathrm{mg} / \mathrm{L}$. The experimental results were shown in Fig. 2. It's obvious from Fig. 2 that the turbidity removal rate of OPSF increased, while that of PFA initially increased and then decreased with an increase of flocculation time. The highest turbidity removal rate of OPSF was $94 \%$ 
at $12 \mathrm{~min}$. The turbidity removal rates of OPSF and PFA were both low which less than $77 \%$ at 2 min. This phenomenon probably stems from the fact that $2 \mathrm{~min}$ flocculation time reduced the effect of co-flocculation. The OPSF optimum flocculation time of 4 min was taken because that there was hardly any change in the curve after $4 \mathrm{~min}$.

In order to investigate the effect of OPSF on removal of turbidity at different sedimentation times, the sedimentation time was taken at 3-13 min and the coagulant dosage still was $6 \mathrm{mg} / \mathrm{L}$. From the information given in Fig. 2(b), we can illustrate that the effect of sedimentation times on the turbidity removal rate of OPSF and PFA were different significantly. The turbidity removal rate of OPSF remained relatively stable which approximately $94 \%$. Therefore, the optimum sedimentation time was $3 \mathrm{~min}$ in view of saving time. The turbidity removal rate of PFA increased sharply at first, then rose slowly and tended to stabilize with the increase of sedimentation time, which was the lowest of less than $47 \%$ at $3 \mathrm{~min}$. The maximum difference between the turbidity removal rate of PFA and OPSF occurred at $3 \mathrm{~min}$, and the turbidity removal rate of OPSF was about $47.1 \%$ higher than that of PFA.
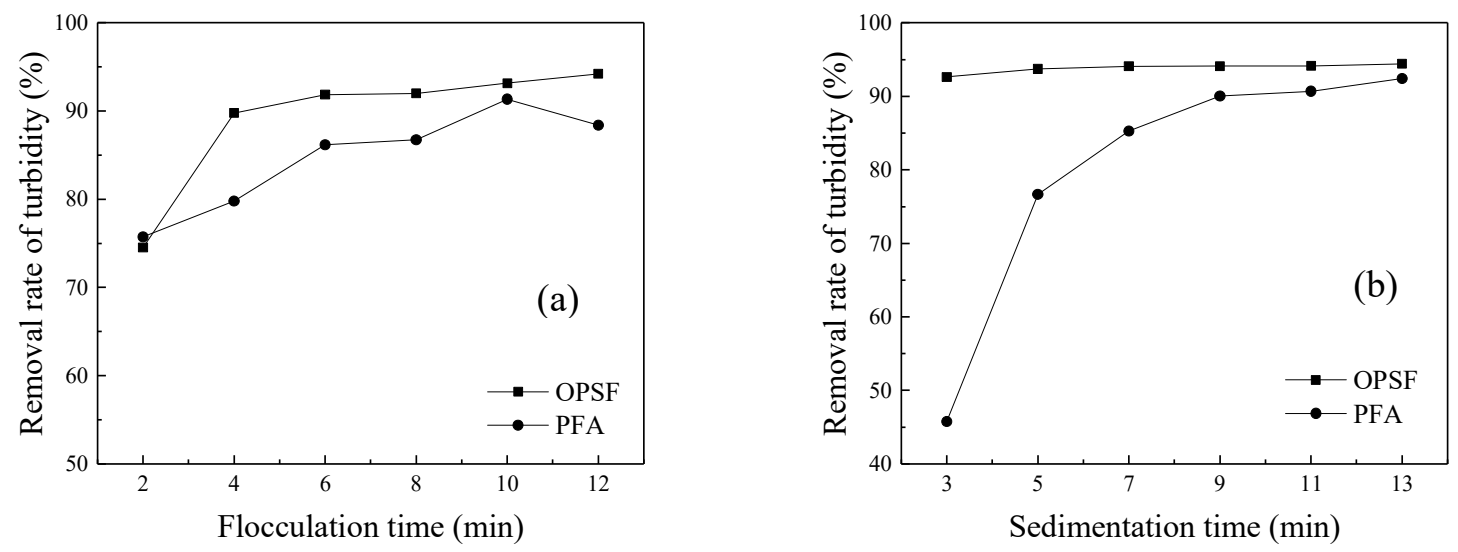

Fig. 2 Effect of flocculation and sedimentation time on the turbidity removal rate of OPSF

Performance and Analysis of OPSF "Short-time Coagulation and Filtration". According to the information gathered above, the experiment was carried out in accordance with above jar tests coagulation step when doing ordinary experiments. But there were some different conditions for short-time coagulation and filtration experiment, as follows: coagulant dosage, flocculation time of and sedimentation time of $6 \mathrm{mg} / \mathrm{L}, 4 \mathrm{~min}$ and $3 \mathrm{~min}$, respectively.

The statistics in Fig. 3 reflected that the turbidity removal rate of OPSF short-term coagulation was roughly consistent with OPSF ordinary coagulation and close to $100 \%$, while the difference was large for PFA. With the increase of filtration time, the turbidity removal rate of PFA had trended decline, among them the short-term coagulation decreased obviously, and the ordinary coagulation decreased slowly. The turbidity of the effluent of the PFA "short-term coagulation and filtration" has dropped to 3.34 NTU when filtered for $2 \mathrm{~h}$, and that of PFA "ordinary coagulation and filtration" was 11.8 NTU at $11 \mathrm{~h}$. They all exceeded the water quality standards in drinking water.

Studies have shown that the OPSF containing ferrosilicon complex had a diastolic and robust

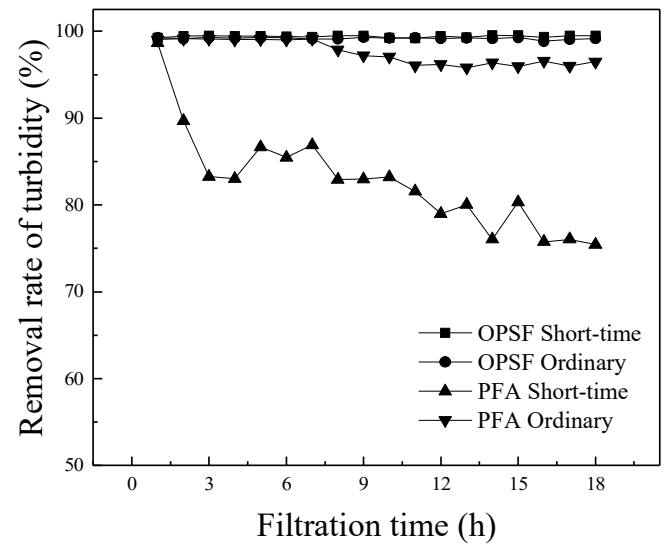

Fig. 3 Comparison of turbidity removal effect of OPSF and PFA "short-time coagulation and filtration" process

structure, and average particle size of OPSF is nearly 11 times than that of PFA [8]. This structure is 
favorable for OPSF to adsorb more particles and bridge to larger and denser flocs. The strong oxidizing ability of OPSF can change the molecular structure of dissolved organic substances, resulting in the hydrophobic property on surface [8], and the adsorption of humic acid particles in water samples was enhanced. Then OPSF can make full use of the adsorption and bridging ability to absorb the hard-to-agglomerate particles in water. Hence, the OPSF short-term coagulation can achieve better treatment effect. When the supernatant after sedimentation passes through the filter layer, a small amount of flocs in the supernatant are almost all adhered and retained by the filter material, so that the filtered effluent showed relatively good water quality. PFA only depends on its electrical neutralization capability owing to its limited bridging capacity, so it cannot form large-size flocs. Therefore, PFA short-term coagulation effect is not ideal, and the supernatant after sedimentation contains more flocs. So that the filtration capacity of the filter layer drops sharply during filtration, and small-size flocs are not retained by the filter material when flowing through the filter layer, leading to poor water quality.

\section{Conclusion}

The optimum dosage of OPSF was $6 \mathrm{mg} / \mathrm{L}$, and the flocculation time of $4 \mathrm{~min}$ had a significant effect on the removal rate of turbidity. With the increase of precipitation time, the removal effect of OPSF on turbidity was relatively stable, and the removal rate remained around $94 \%$. The OPSF short-term coagulation and ordinary coagulation had the same effect on the removal of turbidity, but the PFA was not. And OPSF "short-time coagulation and filtration" was better than PFA "ordinary coagulation and filtration". The OPSF "short-term coagulation and filtration" process had a better treatment effect, which greatly reduced the space and improved the flexibility and applicability of the water-treatment equipment. It provided an experimental and theoretical basis for integrated and modular equipment, and provided a technological basis for solving rural drinking water safety.

\section{Acknowledgements}

This work was financially supported by Shandong higher school science and technology project (L15LG04) and Joint Project between University and Company (W17121).

\section{References}

[1] Y. Fu and S.L. Yu: Desalination Vol. 247 (2009), p. 442

[2] J. Keeley, P. Jarvis, A.D. Smith and S.J. Judd: Water Research Vol. 88 (2016), p. 502

[3] H.Y. Dong, B.Y. Gao, Q.Y. Yue, S.L. Sun, Y. Wang and Q. Li: Environmental Science and Pollution Research Vol. 22 (2015), p. 4566

[4] A.T. Nair and M.M. Ahammed: Journal of Cleaner Production Vol. 96 (2015), p. 272

[5] Y.Q. Chen and W. He: Environmental Engineering Vol. 34 (2016), p. 58

[6] Y. Lu, X. Li, L. Yu and Y. Yang: Water Purification Technology Vol.31 (2012), p. 38

[7] R. Li, J. Pan, W.L.Qin, J. Yang and Y.L. He: Desalination Vol. 351 (2014), p. 37

[8] Y. Fu: Desalination and Water Treatment Vol. 30 (2011), p. 122 